\title{
Acoustic Remote Control of Bacterial Immunotherapy
}

\author{
Mohamad H. Abedi ${ }^{1, \#}$, Michael S. Yao ${ }^{3, \#}$, David R. Mittelstein ${ }^{2}$, Avinoam Bar-Zion ${ }^{3}$, Margaret Swift ${ }^{3}$, \\ Audrey Lee-Gosselin ${ }^{3}$, Mikhail G. Shapiro ${ }^{3, *}$ \\ ${ }^{1}$ Division of Biology and Biological Engineering, California Institute of Technology, Pasadena, CA, USA \\ ${ }^{2}$ Division of Engineering and Applied Sciences, California Institute of Technology, Pasadena, CA, USA \\ ${ }^{3}$ Division of Chemistry and Chemical Engineering, California Institute of Technology, Pasadena, CA, USA \\ \#Equal contribution \\ *Correspondence should be addressed to M.G.S. (mikhail@caltech.edu)
}

\begin{abstract}
Rapid advances in synthetic biology are driving the development of genetically engineered microbes as therapeutic agents for a multitude of human diseases, including cancer. In particular, the immunosuppressive microenvironment of solid tumors creates a favorable niche for systemically administered bacteria to engraft in the tumor and release therapeutic payloads. However, such payloads can be harmful if released in healthy tissues where the bacteria also engraft in smaller numbers. To address this limitation, we engineer therapeutic bacteria to be controlled by focused ultrasound, a form of energy that can be applied noninvasively to specific anatomical sites such as solid tumors. This control is provided by a temperature-actuated genetic state switch that produces lasting therapeutic output in response to briefly applied focused ultrasound hyperthermia. Using a combination of rational design and high-throughput screening we optimized the switching circuits of engineered cells and connected their activity to the release of immune checkpoint inhibitors. In a clinically relevant cancer model, ultrasound-activated therapeutic microbes successfully turned on in situ and induced a marked suppression of tumor growth. This technology provides a critical tool for the spatiotemporal targeting of potent bacterial therapeutics in a variety of biological and clinical scenarios.
\end{abstract}

\section{INTRODUCTION}

Cell therapies are rapidly emerging as an exciting and effective class of technologies for cancer treatment ${ }^{1-3}$. Among the cell types being investigated for therapy, immune cells have excelled in the treatment of hematologic malignancies. However, their use in solid tumors has been hampered by their reduced ability to penetrate and function in the tumor's immunosuppressive environment, especially within immune-privileged hypoxic cores ${ }^{4}$ ${ }^{6}$. Conversely, the reduced immune activity of some tumor cores creates a favorable microenvironment for the growth of certain bacteria, which can reach the tumors after systemic administration $^{7-9}$. Capitalizing on their tumor-infiltrating properties, such bacteria can be engineered to function as effective cellular therapies by secreting therapeutic payloads to directly kill tumor cells or remodel the microenvironment to stimulate antitumor immunity ${ }^{10-15}$. However, the benefits of microbial therapy are often counterbalanced by safety concerns accompanying the systemic injection of microbes into patients with limited control over their biodistribution or activity ${ }^{1,16,17}$. This is especially important given the well-documented engraftment of circulating bacteria into healthy tissues such as the liver, spleen, and certain hypoxic stem cell niches ${ }^{18-21}$. To avoid damaging healthy organs, it is crucial that the therapeutic activity of microbes be targeted to tumors.

Among the available mechanisms to regulate microbial function, systemically administered chemical inducers ${ }^{22,23}$ are incapable of targeting a particular anatomical site. Meanwhile, light-induced control elements provide high spatiotemporal precision $^{24-26}$, but are constrained by the poor penetration of light into intact tissues ${ }^{27}$. In contrast, temperature-based control elements provide a combination of spatiotemporal control and depth, since temperature can be elevated precisely in deep tissues using noninvasive methods such as focused ultrasound (FUS) ${ }^{28-30}$.

Indeed, it was recently demonstrated that FUS can be used in conjunction with temperature-dependent transcription factors to control the expression of bacterial genes ${ }^{31}$. However, these transcription factors operated in therapeutically irrelevant cloning strains of bacteria, had non-therapeutic outputs, and produced only transient activation unsuitable for tumor treatment, which typically requires weeks of therapeutic activity $10,11,22$.

Here we describe the development of FUS-activated therapeutic bacteria in which a brief thermal stimulus activates sustained release of anti-cancer immunotherapy. We engineer these cellular agents by adapting temperature-sensitive transcription factors to the tumor-homing probiotic species $E$. coli Nissle 1917 and designing gene circuits in which they control an integrase-based state switch ${ }^{31,32}$ resulting in long-term therapy production. To improve the safety and efficacy of these cells, we screen random and rationally designed libraries of gene circuit variants for constructs with minimal baseline activity and maximal induction upon thermal stimulation. We use the optimized gene circuits to express immune checkpoint inhibitors targeting CTLA4 and PD-L1. In a mouse cancer model, we show that the resulting engineered microbes are reliably and chronically activated by a brief, noninvasive FUS treatment after systemic administration to release therapy and successfully suppress tumor growth.

\section{RESULTS}

Characterizing thermally responsive repressors in a therapentically relevant microbe. To develop a temperature-actuated therapeutic circuit, we started with highperformance temperature-dependent transcriptional repressors, which actuate transient gene expression in response to small changes in temperature around $37^{\circ} \mathrm{C}^{31}$. Since genetic elements tend to behave differently across cell types due to variations in protein expression and other aspects of the intracellular environment ${ }^{33}$, we first characterized the performance of these repressors in our chosen therapeutic chassis: E. coli Nissle 1917 $(\mathrm{EcN})$. This bacterial strain is approved for human probiotic use and is commonly employed in microbial tumor therapies ${ }^{10,34}$. We selected three repressor candidates - TlpA39, wild-type TcI, and TcI42 - as our starting points due to their desirable activation temperature thresholds of $39^{\circ} \mathrm{C}, 38^{\circ} \mathrm{C}$, and $42^{\circ} \mathrm{C}$, respectively To evaluate the performance of these candidates we designed reporter constructs where they regulate the expression of 
a

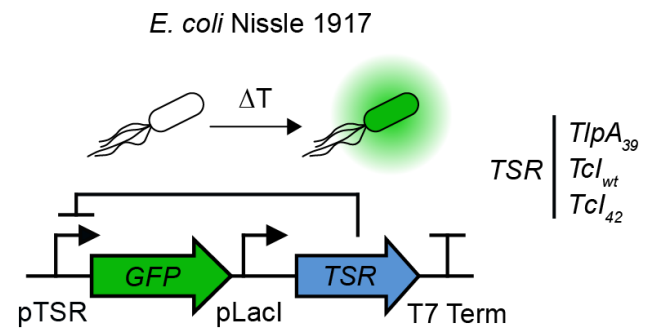

C

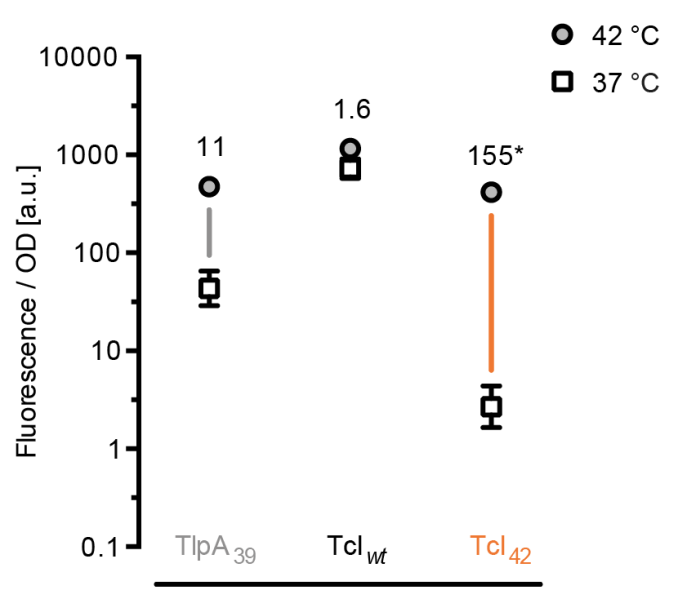

TS repressors

e

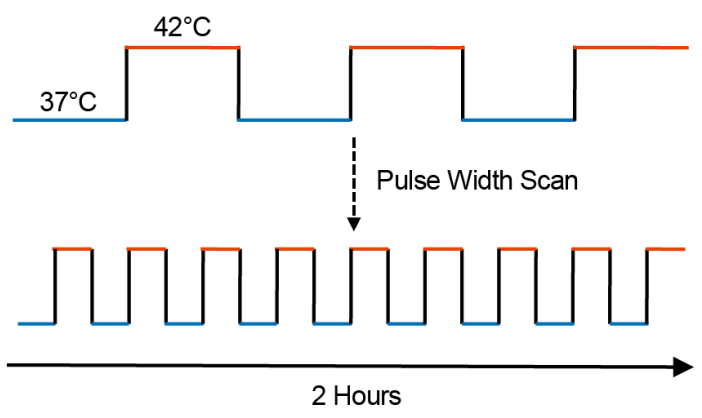

b

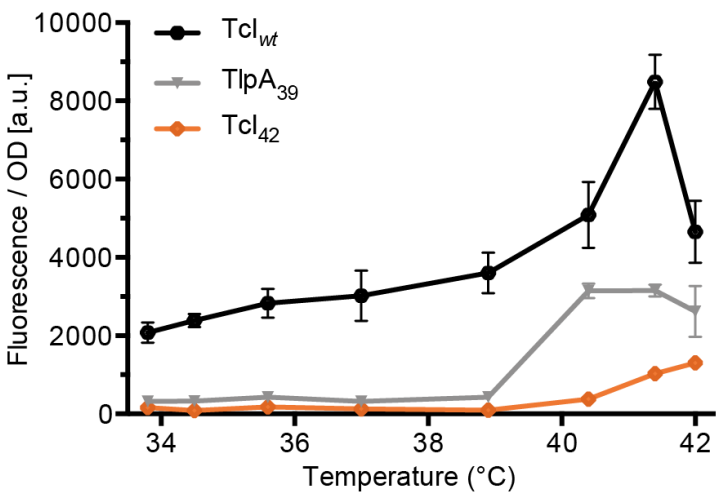

d

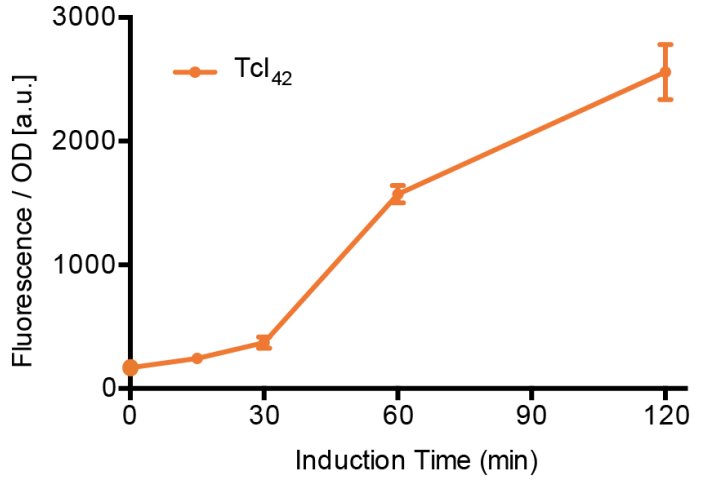

f

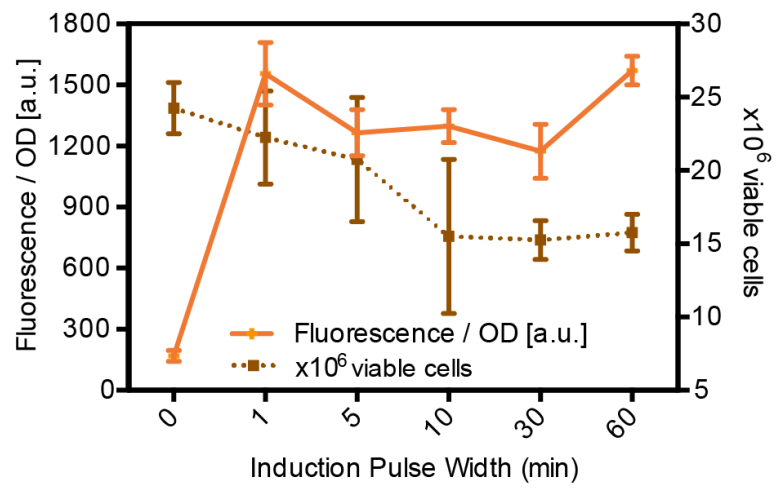

Figure 1 | Evaluating temperature-sensitive transcriptional repressors in $\boldsymbol{E}$. coli Nissle 1917. (a) Illustration of the genetic circuit used to characterize the behavior of temperature-sensitive repressors in E. coli Nissle 1917. (b) Optical density $\left(\mathrm{OD}_{600}\right)$-normalized fluorescence as a function of induction temperature for a fixed duration of 1 hour, measured 24 hours after induction. (c) OD-normalized fluorescence 24 hours after a 1-hour induction at $37^{\circ} \mathrm{C}$ or $42^{\circ} \mathrm{C}$ for the constructs shown in $\mathbf{b}$. Numbers indicate fold-change. (d) OD-normalized fluorescence as a function of induction duration. Cells were stimulated at $42{ }^{\circ} \mathrm{C}$ and fluorescence measured 24 hours later. (e) Illustration of the pulsatile heating scheme used to optimize thermal induction and cell viability. (f) OD-normalized fluorescence as a function of pulse duration for the TcI $\mathrm{I}_{42}$ circuit. All samples were stimulated for a total of 1 hour at $42{ }^{\circ} \mathrm{C}$ and 1 hour at $37{ }^{\circ} \mathrm{C}$ and evaluated 24 hours later. Viable cell counts at various pulse durations plotted to reflect cell viability. Where not seen, error bars $( \pm \mathrm{SEM})$ are smaller than the symbol. $\mathrm{N}=4$ biological replicates for each sample.

a green fluorescent protein $(\mathrm{GFP})($ Fig. 1a), transformed them into EcN cells, and measured the corresponding cell densitynormalized fluorescence intensity as a function of temperature between $33^{\circ} \mathrm{C}$ and $42^{\circ} \mathrm{C}(\mathbf{F i g} . \mathbf{1 b})$. As we were interested in using these bioswitches in vivo, we focused on the fold-change between the mammalian physiological temperature $\left(37^{\circ} \mathrm{C}\right)$ and an elevated temperature that can be used to trigger activation in vivo while minimizing thermal damage to local tissues $\left(42^{\circ} \mathrm{G}\right)$ (Fig. 1c).
Results from these experiments indicated that TcI42 is the best candidate for integration into our thermal switch since it exhibits strong induction at $42{ }^{\circ} \mathrm{C}$ while maintaining low levels of baseline activity.

With TcI42 serving as the thermal transducer in our cells, we next sought to determine the minimal heating duration and ideal heating parameters required to achieve strong activation while minimizing damage to cells. We stimulated cells carrying the 
circuit described in Fig. 1a by elevating the temperature to $42{ }^{\circ} \mathrm{C}$ for different durations and measured the corresponding fluorescence intensity (Fig. 1d). The results indicated that a minimal heating time of one hour is needed for robust activation. We quantified the effect of this thermal dose on microbial cell viability and simultaneously tested a pulsatile heating scheme that was previously shown to enhance viability in mammalian cells ${ }^{35}$. For the pulsatile heating scheme, the duty cycle was kept constant at $50 \%$ while alternating the temperature between $37^{\circ} \mathrm{C}$ and 42 ${ }^{\circ} \mathrm{C}$, resulting in a total of one hour at $42{ }^{\circ} \mathrm{C}$ over a two-hour period, with pulse duration varying between 1 and 60 minutes (Fig. 1e). As hypothesized, cell viability decreased as the pulse duration increased, while induction levels did not significantly vary (Fig. 1f). Based on these results, we selected a five-minute pulse duration for subsequent applications, as this heating paradigm enhanced cell viability while being readily achievable with a focused ultrasound setup. Collectively, our experiments identified and characterized TCI42 as an effective thermal transducer to control gene expression in the therapeutically relevant EcN strain.

Constructing a thermally actuated state switch. On its own, the TcI42 switch is not sufficient for microbial cancer therapy. This switch is transiently activated for the duration of heating, while tumor therapy requires weeks to effectively suppress tumor growth. Since daily FUS application over this period is infeasible in a clinical setting, we set out to engineer a gene circuit that maintains a prolonged therapeutic response following a single, brief thermal activation.

To enable stable thermal switching, we placed the expression of Bxbl, a serine integrase, under the control of a thermally inducible promoter regulated by TcI42 (Fig. 2a). Our design combines the temperature sensitivity of TcI42 with the permanent effector function of the Bxbl integrase. At physiological temperatures of approximately $37^{\circ} \mathrm{C}$, constitutively expressed TcI42 represses the expression of Bxbl. Upon thermal stimulation, the release of TcI42 repression results in a burst of Bxbl expression. Thermally derepressed Bxb1 expression catalyzes the inversion and activation of a $\mathrm{P} 7$ promoter that is responsible for driving the expression of a fluorescent reporter to monitor the state of the circuit and a tetracycline resistance cassette serving as a placeholder for a therapeutic protein (Fig. 2a). Because the DNA inversion event that activates P7 is permanent, this promoter will continue to drive the expression of its protein payloads even when the temperature stimulus is terminated. To avoid unregulated expression of Bxbl we insulated the activity of the temperature-activated promoter by inserting two strong terminators upstream to block activity from other regions of the plasmid $^{36}$.

The ideal performance of the circuit described above would maintain low baseline activity at physiological temperature while providing strong and lasting induction once thermally stimulated. To achieve this performance, we tuned three key sequence elements affecting Bxbl translation and stability: the Bxb1 ribosomal binding sequence (RBS), start codon, and ssrA degradation tag (Fig. 2b). To efficiently identify the best versions of these elements we performed a library screen that consisted of randomized 6-bp sequences within the Bxb1 RBS, two Bxb1 start codon choices, and randomized terminal tripeptides in the Bxb1 ssrA degradation tag ${ }^{37}$. Two start codons were tested because the non-canonical start codon GUG can down-regulate ribosomal efficiency, and the last three amino acids of the ssrA degradation tag were randomized because they strongly modulate the degradation rate of ssrA-tagged proteins ${ }^{38}$. A total landscape of approximately $10^{7}$ possible unique variants was sampled using a high-throughput plate-replication assay (Fig. 2b). Agar plates containing colonies of library members were first replicated, and then one plate was incubated at $37^{\circ} \mathrm{C}$ to assess baseline expression, while the other plate was stimulated at $42{ }^{\circ} \mathrm{C}$ for an hour and returned $37^{\circ} \mathrm{C}$ for the rest of the growth period. The temperature-dependent fluorescence of a representative sampling of variants is shown in Figure 2c. We selected a subset of variants with low leak and high activation to quantify their switching performance with a larger number of replicates $(\mathbf{F i g}$. 2d). Out of these candidates, we selected candidate $\# 5$ for further optimization since it activated the largest percentage of the cells upon stimulation, a metric that is important to ensure strong therapeutic activity in vivo, while still retaining a reasonable temperature-dependent fold change (Fig. 2d).

To reduce the baseline activity of candidate \#5, we modified two additional circuit components (Fig. 2e). The first modification changed the origin of replication from the low-copy origin pSG101 to the medium-copy origin p15A. The second modification explored the effect of inserting a temperaturesensitive terminator upstream of the Bxbl coding sequence. This terminator introduces a temperature-sensitive secondary structure in the mRNA transcript that helps terminate protein expression at low temperatures, adding to the control provided by TcI42 to prevent leaky Bxbl protein production at physiological temperature ${ }^{39}$. At $42{ }^{\circ} \mathrm{C}$, this terminator loses its secondary structure and Bxbl expression is unimpeded. We assessed the performance of four constructs with either one or both of these modifications (Fig. 2f). Increasing the copy number of the plasmid and inserting the terminator reduced baseline activation independently. When combined together, these modifications resulted in significantly reduced leakage while maintaining a large fold-change in activated cells upon induction. The resulting construct, obtained through a combination of randomized and rational engineering, displayed a more than 100 -fold change in activity between $37^{\circ} \mathrm{C}$ and $42^{\circ} \mathrm{C}$.

Engineering cells for thermally actuated secretion of anticancer immunotherapy. To demonstrate that engineered cells containing our optimized thermally actuated circuit can function in a clinically relevant scenario, we modified the output of the circuit to express a therapeutic payload (Fig. 3a). In particular, we selected aCTLA-4 and aPD-L1 nanobodies, which block signaling through the CTLA-4 and PD-L1 checkpoint receptor pathways, that are heavily implicated in T-cell silencing within immunosuppressive solid tumors. Checkpoint inhibitors such as aCTLA-4 and aPD-L1 have emerged as a major class of cancer therapy, but their therapeutic efficacy is commonly accompanied by the risk of unintentionally activating autoimmunity in bystander tissues when administered systemically ${ }^{40,41}$. By combining the ability of FUS to target specific areas deep within tissues with a highly specific thermal switch, we reasoned that we could target the activity of these potent immunomodulators to tumors and thereby mitigate the risk of systemic exposure.

aCTLA-4 and aPD-L1 have been shown to produce antitumor effects when released by tumor-injected probiotics ${ }^{10}$. We hypothesized that local release of these proteins in tumors by FUS-activated systemically administered bacteria would supress tumor growth. To test this hypothesis, we fused aCTLA-4 and aPD-L1 to a PelB secretion tag to enhance their secretion upon 
activation and cloned each construct in place of the tetracycline cassette in our optimized switching circuit. In addition, to stabilize our plasmids for long-term retention in vivo without antibiotic selection, we added an Axe-Txe toxin-antitoxin stability domain, which ensures retention of the plasmid in a cell population by eliminating cells that lose it $^{42,43}$.

The thermal switching functionality of our therapeutic circuits closely resembled their non-therapeutic counterpart. The

a

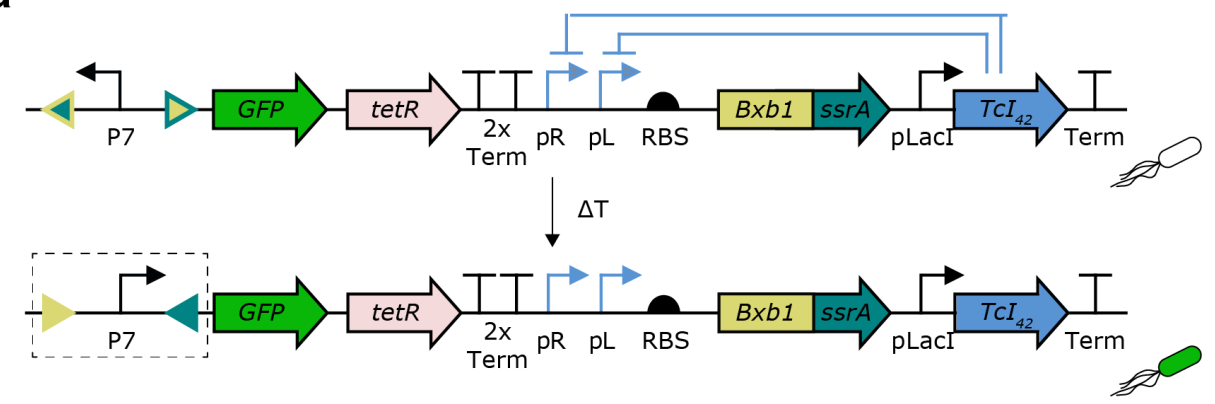

b

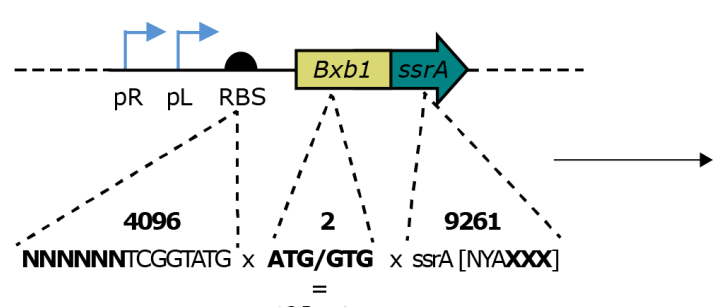

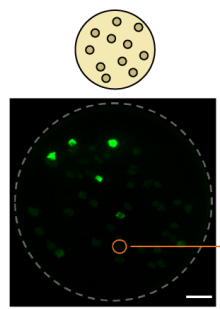

$37^{\circ} \mathrm{C}$

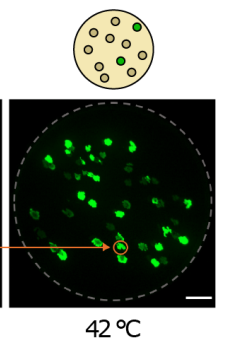

d
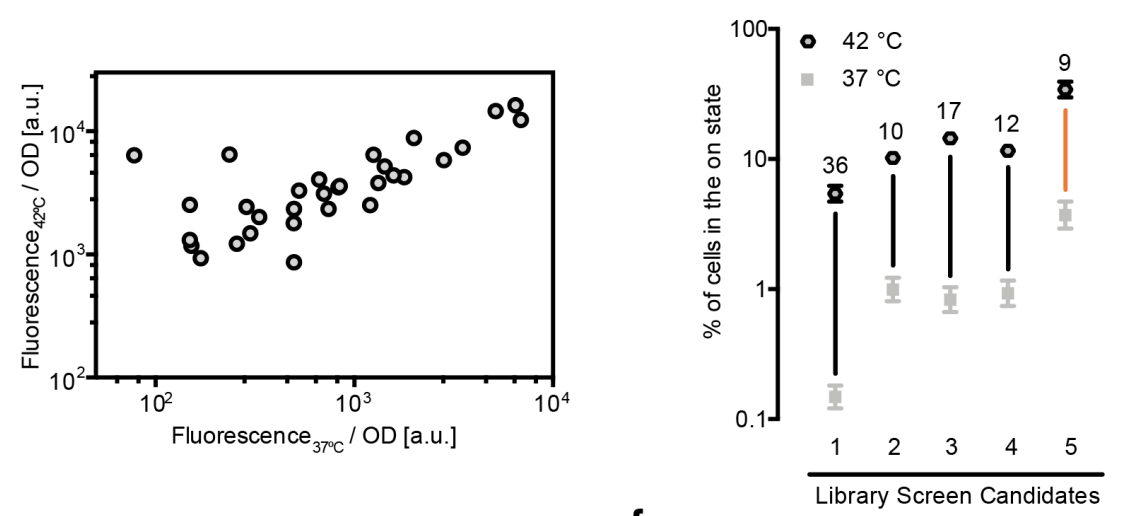

e
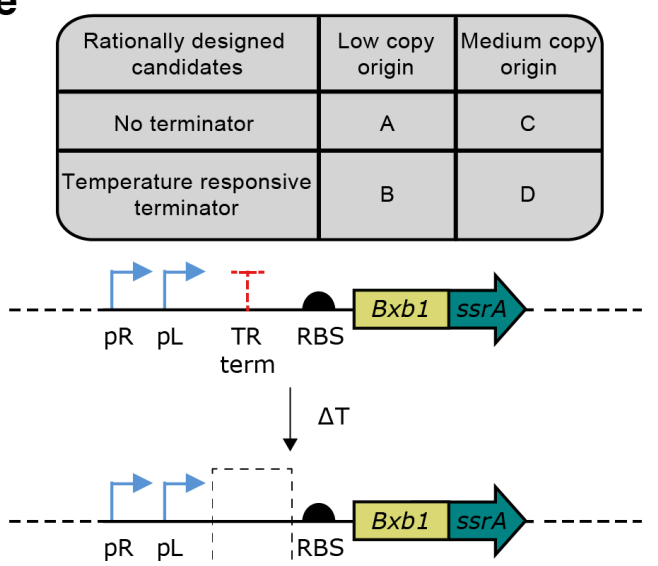

$\mathbf{f}$

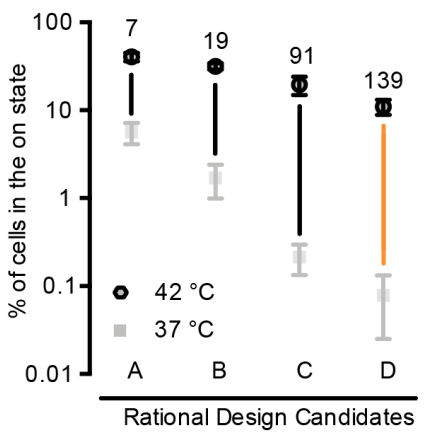

Figure 2 | Construction and optimization of a temperature responsive state switch. (a) Illustration of the genetic circuit constructed to establish a temperature responsive state switch. TetR is the tetracycline resistance cassette. (b) Illustration of the sites targeted in a high throughput screen to optimize circuit switching. A representative fluorescence image of replica plates used to screen for circuit variants. Plates were incubated at the indicated temperature for one hour and further incubated at $37^{\circ} \mathrm{C}$ until colonies grew large enough for analysis. The orange circle indicates an example colony selected for further assay. (c) Circuit variants from the screen in $\mathbf{b}$ characterized for their fluorescence at $37^{\circ} \mathrm{C}$ and $42^{\circ} \mathrm{C}$. (d) Percent conversion to the on-state 24 hours after a 1-hour thermal stimulation at $42{ }^{\circ} \mathrm{C}$ or $37^{\circ} \mathrm{C}$ for five of the circuit variants from $(\mathbf{c})$. (e) Summary of rational modifications made to reduce leakage in the circuit at $37^{\circ} \mathrm{C}$. (f) Percent induction 24 hours after a 1-hour of thermal induction at $42{ }^{\circ} \mathrm{C}$ compared to baseline incubation at $37^{\circ} \mathrm{C}$ for four circuit variants described in e. Error bars represent \pm SEM. N=4 biological replicates for each sample. 
a
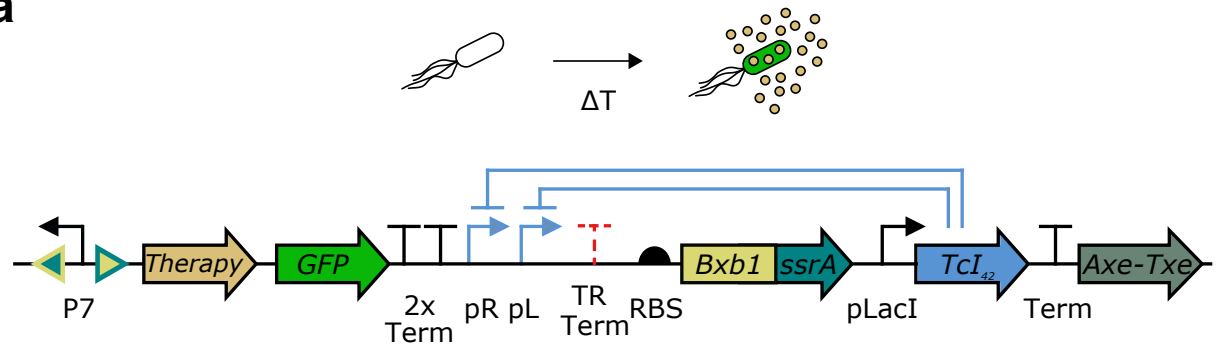

b

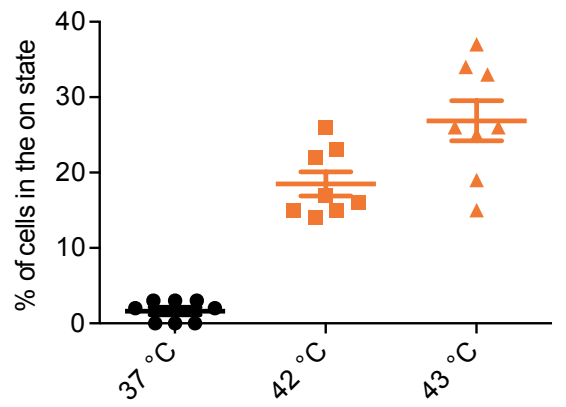

C

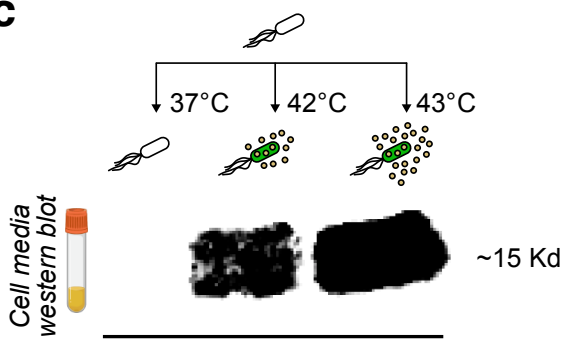

aCTLA-4 released in cell media

Figure 3 | Thermally activated sustained release of immunotherapeutic payload. (a) Temperature responsive state switch modified to release aCTLA-4 or aPD-L1 nanobodies. The circuit includes an Axe-Txe stability cassette. (b) Percent activation 24 hours after a 1-hour of thermal induction at $37^{\circ} \mathrm{C}, 42{ }^{\circ} \mathrm{C}$ or $43^{\circ} \mathrm{C}$ for the circuit described in a. (c) Western blot against hexahistidine-tagged aCTLA-4 nanobodies. Cells were induced for 1 hour at $37^{\circ} \mathrm{C}, 42^{\circ} \mathrm{C}$ or $43^{\circ} \mathrm{C}$, then expanded in $5 \mathrm{ml}$ of media for 24 hours at $37^{\circ} \mathrm{C}$ before collecting the media and assaying for the release of aCTLA-4 nanobodies. The original western blot image is shown in Fig. S1. Similar staining was done to confirm aPD-L1 release.

circuit containing aCTLA-4 maintained a tight off-state at $37^{\circ} \mathrm{C}$ while exhibiting robust fold-changes upon induction at $42^{\circ} \mathrm{C}$ and $43{ }^{\circ} \mathrm{C}$ (Fig. 3b). The $43^{\circ} \mathrm{C}$ condition was added to allow for comparison with in vivo experiments, where this slightly higher temperature is targeted as the focal maximum inside the tumor to allow more of the mass to be heated above $42{ }^{\circ} \mathrm{C}$.

To assess the secretion of therapeutic nanobodies upon activation, we stimulated the cells for one hour at $37^{\circ} \mathrm{C}, 42^{\circ} \mathrm{C}$ and $43{ }^{\circ} \mathrm{C}$, then cultured them for one day at $37{ }^{\circ} \mathrm{C}$, and performed a Western Blot to evaluate the levels of aCTLA-4 nanobodies released in their media. This experiment demonstrated that aCTLA-4 nanobodies are reliably secreted exclusively upon stimulation at $42{ }^{\circ} \mathrm{C}$ and $43{ }^{\circ} \mathrm{C}$ (Fig. 3c). We could not detect any secretion when the cells were incubated at 37

${ }^{\circ} \mathrm{C}$. Similar characterization was performed for cells expressing aPD-L1.

Activation of engineered microbes with focused ultrasound elicits in vivo tumor suppression. To enable thermal control of engineered therapeutic microbes in vivo we built a FUS stimulation setup capable of locally delivering an activation signal within tumors. An ideal setup should be able to elevate the local temperature within a tumor to a predetermined level and autonomously cycle between that temperature and $37^{\circ} \mathrm{C}$ every five minutes to enact our optimized pulsatile heating scheme. In our FUS hyperthermia system (Fig. 4a), a holder secured an anesthetized tumor-bearing mouse vertically in a degassed water chamber. The chamber also held a submerged feedbackcontrolled ultrasound transducer that used the temperature of the tumor to adjust its output intensity in real time to achieve the target temperature. We demonstrated that this system is capable of toggling the temperature in the tumor of a living animal between $37^{\circ} \mathrm{C}$ and $43{ }^{\circ} \mathrm{C}$ every five minutes (Fig. 4a). We set the focal maximum temperature inside the tumor at $43^{\circ} \mathrm{C}$ to allow more of the mass to be heated above $42{ }^{\circ} \mathrm{C}$ and ensure reliable activation within the context of a mouse. While this could lead to some thermal damage, we reasoned that such damage within the tumor is acceptable and could help enhance the microbial therapy ${ }^{44,45}$.

Using this in vivo setup, we tested our ability to locally activate systemically administered therapeutic microbes inside tumors. We seeded $5 \times 10^{6}$ A20 murine tumor cells in the right flanks of BALB/c mice (Fig. 4b). Once the tumors grew to approximately $100 \mathrm{~mm}^{3}$, we intravenously injected $10^{8} \mathrm{EcN}$ cells comprising a 1:1 mixture of cells engineered for thermallyactuated aCTLA-4 or aPD-L1 secretion. Injected microbes were given two days to engraft in tumors before they were stimulated with FUS. After FUS activation, tumor growth was monitored to assess therapeutic efficacy.

We observed major retardation in tumor growth in FUStreated tumors colonized by therapeutic cells, while growth rates in controls including non-FUS treated mice, animals treated with only FUS, and subjects injected with wild-type $\mathrm{EcN}$ were substantially higher (Fig. 4c). After completing this experiment, we collected the tumors, chemically homogenized them, and plated the suspension on selective media. By counting the percentage of activated bacteria, we demonstrated that our thermal switch is exclusively activated in targeted tumors and remains active up to at least two weeks post activation (Fig. 4d). One of the six FUS-activated tumors disappeared as a result of the treatment and bacterial activation inside it could not be quantified. In two out of six FUS-treated tumors, ultrasound failed to activate the therapeutic bacterial circuit. This could be due to limitations in our heating setup, which is currently capable of only partially heating the tumor mass. The two non-activated mice were removed from our analysis of tumor growth. Overall, our in vivo experiments demonstrated that EcN cells engineered for thermally controlled checkpoint inhibition could home to and engraft in tumors from systemic circulation, become activated specifically in response to FUS, maintain this activity for at least two weeks after a 1-hour FUS treatment and significantly reduce tumor growth. 
a

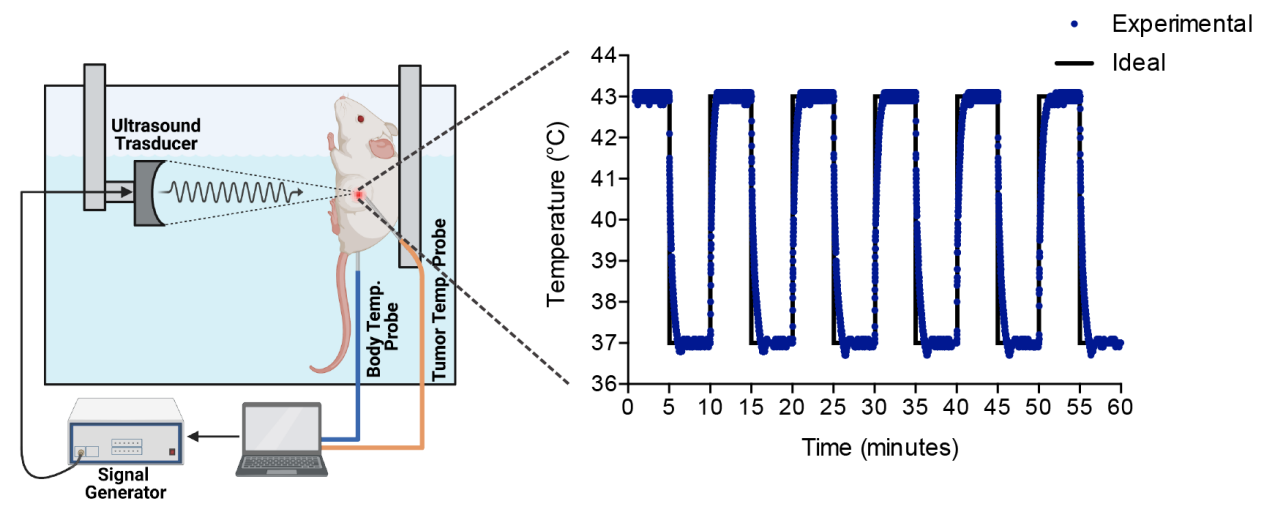

b

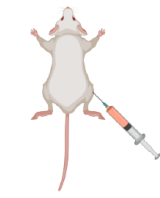

A20 tumors injected

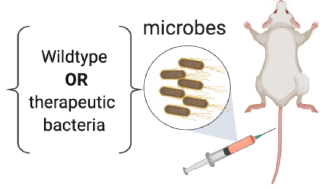

Microbes injected when tumors reach $\sim 100 \mathrm{~mm}^{3}$

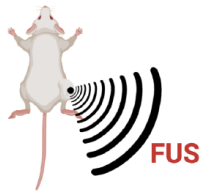

Ultrasound thermal activation

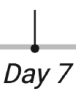

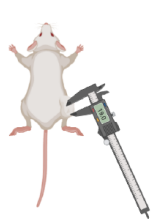

Tumors measured until end point $\left(>1000 \mathrm{~mm}^{3}\right.$ )

Day 9

C

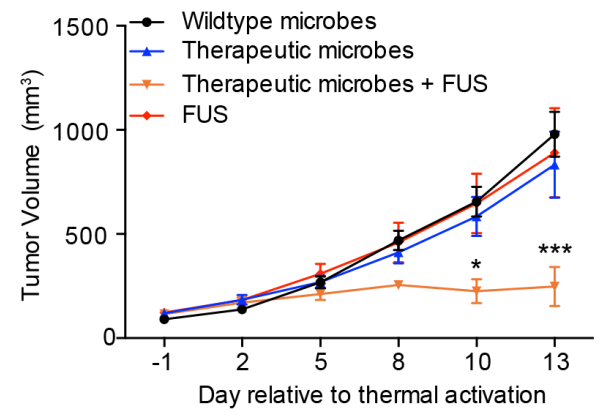

d

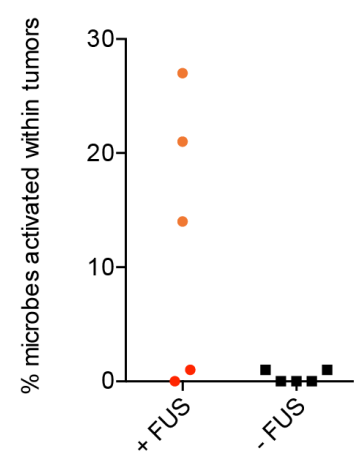

Figure 4 | Ultrasound-activated bacterial immunotherapy reduces tumor growth in vivo. (a) Illustration of the automated setup used to deliver FUS hyperthermia to tumors (left) and representative time course of tumor temperature from a mouse treated with alternating 5-min steps between $37^{\circ} \mathrm{C}$ and $43^{\circ} \mathrm{C}$. (b) Diagram illustrating the experiment performed to assess the activation of microbial antitumor immunotherapy in vivo. Mice were injected with a 1:1 mixture of EcN cells carrying the aCTLA-4 or aPD-L1 circuits, or wildtype EcN. EcN cells were washed and adjusted to $0.625 \mathrm{OD}_{600}$ before injecting $100 \mu \mathrm{L}$ per mouse intravenously. Ultrasound was applied for a total of 1 hour at $43^{\circ} \mathrm{C}$ with $50 \%$ duty cycle and 5 -min pulse duration. (c) Tumor sizes measured over two weeks in mice treated with wildtype EcN, therapeutic microbes in the absence of FUS, therapeutic microbes and FUS treatment, or FUS treatment alone. (d) Percent activation of therapeutic EcN isolated from FUS-treated and non-FUS-treated tumors two weeks after FUS treatment. One of the FUS-activated tumors disappeared after treatment and bacterial activation inside it could not be quantified. Where not seen, error bars $( \pm \mathrm{SEM})$ are smaller than the symbol. At least five mice were used for each control condition.

\section{DISCUSSION}

Our results establish a cell-based system for targeted immunotherapy that couples the special ability of therapeutic bacteria to home into the necrotic core of solid tumors with the capacity of FUS to locally activate their therapeutic function. The sustained activation of these therapeutic bacteria is enabled by a thermal state switch developed through high throughput genetic engineering to have low baseline activity, rapid induction upon stimulation and sustained activity in situ. When this state switch is used to actuate the release of immune checkpoint inhibitors, the resulting engineered microbes can be activated inside tumors by brief FUS exposure to secrete their therapeutic payload over an extended timeframe and substantially reduce tumor growth.

The growing body of work on bacteria-based therapies ${ }^{46-}$ ${ }^{49}$ and the increasing clinical acceptance of FUS ${ }^{50,51}$ provide FUSactuated bacterial therapeutics a path to ultimate clinical implementation. Potential disease targets include cancers with readily identified primary masses that are challenging to resect surgically, such as head-and-neck, ovarian, pancreatic or brain tumors. FUS-actuated bacterial therapeutics could be also relevant to metastatic tumors since microbial therapy in a single tumor mass can generate a strong systemic adaptive immune response, and eliminate distant tumor lesions through a potent abscopal effect $^{52}$. However, further work will be needed to optimize the timing, dose and molecular identity of FUS-activated therapy release for each application. To enhance therapeutic efficacy, it may be beneficial to combine FUS-activated bacterial therapeutics with other molecular or cellular therapies. For example, engineered bacteria and immune cells have distinct and often complementary tumor entry and engraftment profiles. Engineering microbes that successfully enter immunosuppressed tumor regions to secrete checkpoint inhibitors or cytokines could help make this environment more accessible to engineered T cells. In this way, the bacteria and $\mathrm{T}$ cells can synergistically exert their 
therapeutic function from the inside-out and from the outside-in, respectively. Beyond tumor therapy, locally activated bacterial agents have potential utility in a wide array of other biomedical applications. For example, FUS-controlled state switches could be useful in controlling the activity of gut microbes in vivo $0^{53}$, the function of cell-based living materials in vitro ${ }^{54-57}$, and in industrial metabolic engineering ${ }^{24,58}$.

\section{MATERIALS AND METHODS}

\section{Plasmid Construction and Molecular Biology}

All plasmids were designed using SnapGene (GSL Biotech) and assembled via reagents from New England Biolabs for KLD mutagenesis (E0554S) or Gibson Assembly (E2621L). After assembly, constructs were transformed into NEB Turbo (C2984I) and NEB Stable (C3040I) E. coli for growth and plasmid preparation. The Bxb1 recombinase-encoding gene was a kind gift of Richard Murray (Caltech). Integrated DNA Technologies synthesized other genes and all PCR primers. Plasmids containing the aCTLA-4, aPD-L1, and Axe-Txe genes were kind gifts from of Tal Danino (Columbia University).

\section{Preparation of cell lines for in vitro and in vivo experiments}

Plasmids containing engineered genetic circuits were transformed into Nissle 1917 E. coli (Mutaflor $\left.{ }^{\circledR}\right)$. Nissle cells were cultured in LB broth (Sigma) and grown on LB agar plates (Sigma) containing appropriate antibiotics. Singular colonies were picked into LB broth and grown overnight in a shaking incubator $\left(30^{\circ} \mathrm{C}, 250\right.$ $\mathrm{rpm})$. The next day, optical density measurements $\left(\mathrm{OD}_{600}\right)$ were taken, and the saturated cultures were diluted to $0.1 \mathrm{OD}_{600}$. Diluted cultures were then allowed to grow to exponential phase until they reached $0.6 \mathrm{OD}_{600}$ before starting assays. Optical density measurements were taken using a Nanodrop 2000c (Thermo Scientific) in cuvette mode.

\section{Western Blot}

Five milliliters of cell media were collected for each sample and concentrated with an Amicon ${ }^{\circledR}$ Ultra-15 Centrifugal Filter Unit. Concentrated cell media was then mixed with Laemmli loading buffer and BME before loading into a pre-cast polyacrylamide gels SDS-PAGE gel (Bio Rad) and ran at $75 \mathrm{~V}$ for 140 minutes. Western blotting was performed using the Transblot Turbo apparatus and nitrocellulose membrane kit (Bio Rad). Transfer was performed at $25 \mathrm{~V}$ for 7 minutes. Membranes were blocked with 5\% Blotto milk (Santa Cruz Biotechnology) in 0.05\% TBSTween for 1 hour at room temperature. Primary staining was performed using the mouse anti-His sc-8036 antibody (Santa Cruz Biotech) overnight at $4^{\circ} \mathrm{C}$. Blots were then washed three times for 15 minutes at $4{ }^{\circ} \mathrm{C}$ with $0.05 \%$ TBS-Tween and stained for 4 hours with mouse IgG kappa binding protein (m-IgGk BP) conjugated to Horseradish Peroxidase (HRP) (Santa Cruz Biotech, sc-516102) at room temperature. After three 15-minute washes, HRP visualization was performed using Super signal west Pico PLUS reagent (Thermo Fisher Scientific). Imaging was performed in a Bio-Rad ChemiDoc MP gel imager. A subsequent epi white light image of the blot under the same magnification was acquired to visualize the stained molecular weight standards.

\section{Thermal regulation assay}

Once bacterial cell cultures reached approximately $0.6 \mathrm{OD}_{600}, 50$ $\mu \mathrm{L}$ aliquots of each sample was transferred into individual Bio-Rad PCR strips with optically transparent caps and subsequently heated in conditions specific to the experiment using a Bio-Rad G100 Touch thermocycler with the lid set to $50{ }^{\circ} \mathrm{C}$. Following heating, cells continued to incubate overnight undisturbed at either $30^{\circ} \mathrm{C}$ (Figure 1) or $37^{\circ} \mathrm{C}$ (Figure 2-4). The PCR strips were then removed, vortexed, and spun down, and the green fluorescence of each of the samples was measured using the Strategene MX3005p qPCR (Agilent) and an unamplified FAM filter. To measure cell density, the samples were diluted $1: 4$ with fresh LB media (without antibiotic) and then transferred into individual wells of a 96-well plate (Costar black/clear bottom). Optical density measurements were taken using the SpectraMax M5 plate reader (Molecular Devices). In order to quantify the temperature-dependent gene expression $(E)$ using backgroundsubtracted, OD-normalized fluorescence (Fig. 1b-d, 1f, 2c), Equation (1) was used:

$$
E=\frac{F_{\text {sample }}-F_{\text {blank }}}{O D_{\text {sample }}-O D_{\text {blank }}}
$$

In this equation, we define $F$ as the raw fluorescence measurement and $O D$ is the $\mathrm{OD}_{600}$ measurement of the sample. The value of the blank fluorescence and blank optical density was determined as the average of $\mathcal{N}=4$ samples of untransformed Nissle cells, as opposed to engineered Nissle cells, in LB.

\section{Screens to optimize circuit behavior}

To improve Bxbl thermal regulation, a sequence randomized library of the RBS, start codon, and ssrA degradation tag was ordered from Integrated DNA Technologies. PCR products that included the Bxbl coding region and immediately surrounding sequences were amplified using custom primers and were inserted into the backbone of the rest of the parent plasmid using Gibson Assembly (Fig. 2b-d). This library was transformed into EcN and plated on LB Agar plates with antibiotic resistance at a low colony density of approximately 30 colonies per petri dish. Following overnight incubation at $30^{\circ} \mathrm{C}$ to allow the colonies to become visible, these plates were then replicated into two daughter petri dishes using a replica-plating tool (VWR 25395-380). The parent petri dish was incubated at $4{ }^{\circ} \mathrm{C}$ until the conclusion of the experiment. One daughter plate was grown overnight at the baseline temperature of $37^{\circ} \mathrm{C}$, and the other was incubated at 42 ${ }^{\circ} \mathrm{C}$ for 1 hour and then moved to $37^{\circ} \mathrm{C}$ overnight. After colonies became visible, the plates were imaged using a 530/28 nm emission filter to determine colonies that were fluorescent at the 'on' temperature but opaque at the 'off' temperature (Bio-Rad ChemiDoc MP imager). Promising library variants were then picked from the corresponding parent petri dish at $4{ }^{\circ} \mathrm{C}$ and analysed against the parent plasmid of the library using the liquid culture fluorescence-based assay described above.

\section{Percent switching assay}

Strips of liquid bacteria samples were prepared and incubated in the Bio-Rad Touch thermocycler. After the prescribed thermal stimulus and incubation at $37^{\circ} \mathrm{C}, \mathrm{PCR}$ strips were removed, vortexed, and spun down on a tabletop centrifuge. Five 1:10 serial dilutions in liquid LB were then performed, transferring $10 \mu \mathrm{L}$ of sample into $90 \mu \mathrm{L}$ of LB media sequentially. After thorough mixing, $50 \mu \mathrm{L}$ of the most diluted samples was plated onto an LB plate and allowed to incubate at $30{ }^{\circ} \mathrm{C}$ overnight. Upon the appearance of visible colonies, plates were imaged using the same Bio-Rad ChemiDoc MP imager with both blue epifluorescence illumination and the 530/28 nm emission filters. The percentage 
of colonies in the 'on state' $(P)$ was determined according to Equation (2):

$$
P=\frac{\text { number of green colonies counted on a plate }}{\text { total number of colonies counted on a plate }}
$$

\section{Animal procedures}

All animal procedures were performed under a protocol approved by the California Institute of Technology Institutional Animal Care and Use Committee (IACUG). 8-12 week-old BALB/c female mice were purchased from Jackson Laboratory. To establish A20 tumor models in mice, $5 \times 10^{6}$ A20 cells were collected and suspended in $100 \mu \mathrm{L}$ phosphate buffer saline (PBS) prior to subcutaneous injection into the flank of each mouse. When tumor volumes reached approximately $100 \mathrm{~mm}^{3}$, engineered EcN cells prepared according to the procedure outlined in the section above were then collected by centrifugation (3000 $\mathrm{g}$ for $5 \mathrm{~min}$ ), washed with phosphate buffer saline PBS 3 times, and diluted in PBS to $0.625 \mathrm{OD}_{600} .100 \mu \mathrm{L}$ of the resulting solution was injected into each of the A20 tumor bearing mice via tail vein. For thermal actuation using ultrasound, mice were anesthetized using a $2 \%$ isoflurane-air mixture and placed on a dedicated animal holder. Anesthesia was maintained over the course of the ultrasound procedure using $1-1.5 \%$ isoflurane, adjusted in real-time to maintain the respiration rate at 20-30 breaths per minute. Body temperature was continuously monitored using a fiber optic rectal thermometer (Neoptix). When appropriate, the target flank was thermally activated using the automated FUS setup described below, cycling between the temperatures of $43^{\circ} \mathrm{C}$ and $37^{\circ} \mathrm{C}$ every 5 minutes for 1 hour of total heating. Following ultrasound treatment, the mouse was returned to its cage and the size of its tumor was measured with a caliper to track the therapeutic efficacy. When the tumors reached $\sim 1000 \mathrm{~mm}^{3}$ mice were culled and the tumors were collected for analysis. Mice that did not have microbial cells in their tumors were excluded from the study.

\section{Tumor analysis}

Tumors were collected and homogenized in ten milliliters of PBS containing $2 \mathrm{mg} / \mathrm{ml}$ collagenase and $0.1 \mathrm{mg} / \mathrm{ml}$ DNAse for one hour at $37{ }^{\circ} \mathrm{C}$. Homogenized tumors were serially diluted and plated onto LB plates to quantify the number of cells colonizing the tumors. The percentage of cells activated within tumors was determined by counting the number of GFP positive cells.

\section{Feedback-controlled focused ultrasound}

We developed a closed loop thermal control setup to maintain a specified predetermined temperature within the tumor of a mouse by modulating the intensity of the FUS. This setup includes a water bath filled with pure distilled water that is being actively cleaned and degassed with an AQUAS-10 water conditioner (ONDA) and maintained at $33{ }^{\circ} \mathrm{C}$ with a sous vide immersion cooker (InstantPot Accu Slim). A tumor-bearing mouse that has been anesthetized as described above is fastened nose up vertically to an acrylic arm that is connected to a manual 3D positioning system (Thorlabs) to enable 3D motion of the mouse within the water bath. A Velmex BiSlide motorized positioning system is used to submerge and position the 0.67 MHz FUS transducer (Precision Acoustics PA717) such that the focal point of the transducer lies within the tumor of the mouse. A signal generator (B\&K \#4054B) generates the thermal ultrasound signal which is then amplified (AR \#100A250B) and sent to drive the ultrasound transducer. The water in this chamber acts as the coupling medium to transfer the ultrasound wave from the transducer to the tumor. To measure the internal tumor temperature during a heating session we temporarily implant a thin fiber optic temperature probe (Neoptix) into the tumors. This temperature readout is also used to align the focus of the transducer with the tumor by emitting a constant test thermal ultrasound signal. Once the system is aligned, we run a Matlab closed loop thermal control script that regulates the signal generator output. Feedback for the controller is provided by the temperature measurements acquired with a sampling rate of $4 \mathrm{~Hz}$. The actuator for the controller is the voltage amplitude of the continuous sinusoidal signal at $0.67 \mathrm{MHz}$ used to drive the FUS transducer, where the voltage is adjusted also at $4 \mathrm{~Hz}$. The system uses a PID controller with anti-windup control that modifies the amplitude of the thermal ultrasound waveform to achieve a desired temperature in the targeted tissues. The $\mathrm{Kp}, \mathrm{Ki}, \mathrm{Kd}$, and $\mathrm{Kt}$ parameters for the PID and anti-windup were tuned using Ziegler-Nichols method, and in some cases adjusted further through trial-and-error tuning to achieve effective thermal control.

\section{Statistics and replicates}

Data is plotted and reported in the text as the mean \pm S.E.M. Sample size is $\mathcal{N}=4$ biological replicates in all in vitro experiments unless otherwise stated. This sample size was chosen based on preliminary experiments indicating that it would be sufficient to detect significant differences in mean values. $P$ values were calculated using a two-tailed unpaired $t$-test.

\section{Data and code availability}

Plasmids will be made available through Addgene upon publication. All other materials and data are available from the corresponding author upon reasonable request.

\section{ACKNOWLEDGEMENTS}

The authors thank Tal Danino, Tiffany Chien, Candice Gurbatri, Sreyan Chowdhury, Dan Piraner and Victoria Hsiao for sharing reagents and helpful discussions. Figure $4 \mathrm{~b}$ was created with BioRender.com. This research was funded by the Sontag Foundation, the Army Institute for Collaborative Biotechnologies (W911NF-19-D-0001) and the Defence Advanced Research Projects Agency (D14AP00050). M.H.A. was supported by the NSF graduate research fellowship and the Paul and Daisy Soros Fellowship for New Americans. AB-Z was supported by the European Union's Horizon 2020 research and innovation programme under the Marie Skłodowska-Curie grant agreement No. 792866. Related research in the Shapiro laboratory is supported by the Burroughs Welcome Career Award at the Scientific Interface, the Packard Foundation Fellowship in Science and Engineering, the Pew Scholarship in the Biomedical Sciences and the Heritage Medical Research Institute.

\section{AUTHOR CONTRIBUTIONS}

M.H.A. and M.G.S. conceived the study. M.H.A., M.S.Y, D.R.M, M.S and AL-G planned and performed experiments. D.R.M. wrote the MATLAB script for in vivo thermal control. AB-Z and D.R.M helped with building the ultrasound heating setup. M.H.A. and M.S.Y analysed data. M.H.A., M.S.Y, D.R.M and M.G.S. wrote the manuscript with input from all other authors. M.G.S. supervised the research.

\section{COMPETING INTERESTS}

The authors declare no competing financial interests. 


\section{REFERENCES}

(1) Zhou, S., Gravekamp, C., Bermudes, D., and Liu, K. (2018) Tumour-targeting bacteria engineered to fight cancer. Nature Reviewes Cancer 18, 727-743.

(2) Weber, E. W., Maus, M. V., and Mackall, C. L. (2020) The Emerging Landscape of Immune Cell Therapies. Cell 181, 46-62.

(3) Yu, J. X., Upadhaya, S., Tatake, R., Barkalow, F., and Hubbard-Lucey, V. M. (2020) Cancer cell therapies: the clinical trial landscape. Nature Reviewes Drug Discovery 19, 583-584.

(4) Fucà, G., Reppel, L., Landoni, E., Savoldo, B., and Dotti, G. (2020) Enhancing Chimeric Antigen Receptor T-Cell Efficacy in Solid Tumors. Clinical Cancer Research 26, 2444-2451.

(5) Anderson, K. G., Stromnes, I. M., and Greenberg, P. D. (2017) Obstacles posed by the tumor microenvironment to T cell activity: a case for synergistic therapies. Cancer Cell 31, 311-325.

(6) Mirzaei, H. R., Rodriguez, A., Shepphird, J., Brown, C. E., and Badie, B. (2017) Chimeric Antigen Receptors T Cell Therapy in Solid Tumor: Challenges and Clinical Applications. Front. Immunol. 8.

(7) Dang, L. H., Bettegowda, C., Huso, D. L., Kinzler, K. W., and Vogelstein, B. (2001) Combination bacteriolytic therapy for the treatment of experimental tumors. Proc Natl Acad Sci U S A 98, 15155-15160.

(8) Leschner, S., Westphal, K., Dietrich, N., Viegas, N., Jablonska, J., Lyszkiewicz, M., Lienenklaus, S., Falk, W., Gekara, N., Loessner, H., and Weiss, S. (2009) Tumor Invasion of Salmonella enterica Serovar Typhimurium Is Accompanied by Strong Hemorrhage Promoted by TNF-a. PLoS One 4.

(9) Kang, S.-R., Jo, E. J., Nguyen, V. H., Zhang, Y., Yoon, H. S., Pyo, A., Kim, D.-Y., Hong, Y., Bom, H.-S., and Min, J.-J. (2020) Imaging of tumor colonization by Escherichia coli using 18F-FDS PET. Theranostics 10, 4958-4966.

(10) Gurbatri, G. R., Lia, I., Vincent, R., Coker, C., Castro, S., Treuting, P. M., Hinchliffe, T. E., Arpaia, N., and Danino, T. (2020) Engineered probiotics for local tumor delivery of checkpoint blockade nanobodies. Science Translational Medicine 12.

(11)Jiang, S.-N., Phan, T. X., Nam, T.-K., Nguyen, V. H., Kim, H.-S., Bom, H.-S., Choy, H. E., Hong, Y., and Min, J.J. (2010) Inhibition of Tumor Growth and Metastasis by a Combination of Escherichia coli-mediated Cytolytic Therapy and Radiotherapy. Molecular Therapy 18, 635-642.

(12) Ryan, R. M., Green, J., Williams, P. J., Tazzyman, S., Hunt, S., Harmey, J. H., Kehoe, S. C., and Lewis, C. E. (2009) Bacterial delivery of a novel cytolysin to hypoxic areas of solid tumors. Gene Therapy 16, 329-339.

(13) Groot, A. J., Mengesha, A., Wall, E. van der, Diest, P. J. van, Theys, J., and Vooijs, M. (2007) Functional antibodies produced by oncolytic clostridia. Biochemical and Biophysical Research Communications 364, 985-989.

(14) Duong, M. T.-Q., Qin, Y., You, S.-H., and Min, J.-J. (2019) Bacteria-cancer interactions: bacteria-based cancer therapy. Experimental \& Molecular Medicine 51, 1-15.

(15) Coley, W. B. (1891) II. Contribution to the Knowledge of Sarcoma. Ann Surg 14, 199-220.

(16) Felgner, S., Pawar, V., Kocijancic, D., Erhardt, M., and Weiss, S. (2017) Tumour-targeting bacteria-based cancer therapies for increased specificity and improved outcome. Microbial Biotechnology 10, 1074-1078.

(17) Chien, T., Harimoto, T., Kepecs, B., Gray, K., Coker, C., Pu, K., Azad, T., and Danino, T. (2019) Multiplexed biosensors for precision bacteria tropism in vivo. bioRxiv 851311.

(18) Clairmont, G., Lee, K. C., Pike, J., Ittensohn, M., Low, K. B., Pawelek, J., Bermudes, D., Brecher, S. M., Margitich, D., Turnier, J., Li, Z., Luo, X., King, I., and Zheng, L. M. (2000) Biodistribution and Genetic Stability of the Novel Antitumor Agent VNP20009, a Genetically Modified Strain of Salmonella typhimuvium. The Journal of Infectious Diseases 181, 1996-2002.

(19) Park, S.-H., Zheng, J. H., Nguyen, V. H., Jiang, S.-N., Kim, D.-Y., Szardenings, M., Min, J. H., Hong, Y., Choy, H. E., and Min, J.-J. (2016) RGD Peptide Cell-Surface Display Enhances the Targeting and Therapeutic Efficacy of Attenuated Salmonella-mediated Cancer Therapy. Theranostics 6, 1672-1682.

(20) Stritzker, J., Weibel, S., Hill, P. J., Oelschlaeger, T. A., Goebel, W., and Szalay, A. A. (2007) Tumor-specific colonization, tissue distribution, and gene induction by probiotic Escherichia coli Nissle 1917 in live mice. International Fournal of Medical Microbiology 297, 151-162.

(21) Massa, P. E., Paniccia, A., Monegal, A., de Marco, A., and Rescigno, M. (2013) Salmonella engineered to express CD20-targeting antibodies and a drug-converting enzyme can eradicate human lymphomas. Blood 122, 705-714.

(22) Zheng, J. H., Nguyen, V. H., Jiang, S.-N., Park, S.-H., Tan, W., Hong, S. H., Shin, M. G., Chung, I.-J., Hong, Y., Bom, H.-S., Choy, H. E., Lee, S. E., Rhee, J. H., and Min, J.-J. (2017) Two-step enhanced cancer immunotherapy with engineered Salmonella typhimurium secreting heterologous flagellin. Science Translational Medicine 9. 
(23) Dai, Y., Toley, B. J., Swofford, C. A., and Forbes, N. S. (2013) Construction of an inducible cell-communication system that amplifies Salmonella gene expression in tumor tissue. Biotechnology and Bioengineering 110, 1769-1781.

(24) Hartsough, L. A., Park, M., Kotlajich, M. V., Lazar, J. T., Han, B., Lin, G.-C. J., Musteata, E., Gambill, L., Wang, M. C., and Tabor, J. J. (2020) Optogenetic control of gut bacterial metabolism to promote longevity. eLife (Gruber, J., Tyler, J. K., and Mair, W., Eds.) 9, e56849.

(25) Lalwani, M. A., Ip, S. S., Carrasco-López, C., Day, C., Zhao, E. M., Kawabe, H., and Avalos, J. L. (2021) Optogenetic control of the lac operon for bacterial chemical and protein production. Nature Chemical Biology 17, 71-79.

(26) Liu, Z., Zhang, J., Jin, J., Geng, Z., Qi, Q., and Liang, Q. (2018) Programming Bacteria With Light-Sensors and Applications in Synthetic Biology. Front Microbiol 9.

(27) Ash, C., Dubec, M., Donne, K., and Bashford, T. (2017) Effect of wavelength and beam width on penetration in light-tissue interaction using computational methods. Lasers Med Sci 32, 1909-1918.

(28) Rome, C., Couillaud, F., and Moonen, C. T. W. (2005) Spatial and temporal control of expression of therapeutic genes using heat shock protein promoters. Methods 35, 188-198.

(29) Moonen, C. T. W. (2007) Spatio-Temporal Control of Gene Expression and Cancer Treatment Using Magnetic Resonance Imaging-Guided Focused Ultrasound. Clin Cancer Res 13, 3482-3489.

(30) Kruse, D. E., Mackanos, M. A., O\textquotesingleConnell-Rodwell, G. E., Contag, C. H., and Ferrara, K. W. (2008) Short-duration-focused ultrasound stimulation of Hsp70 expressionin vivo. Phys. Med. Biol. 53, 3641-3660.

(31) Piraner, D. I., Abedi, M. H., Moser, B. A., Lee-Gosselin, A., and Shapiro, M. G. (2017) Tunable thermal bioswitches for in vivo control of microbial therapeutics. Nature Chemical Biology 13, 75-80.

(32) Hsiao, V., Hori, Y., Rothemund, P. W., and Murray, R. M. (2016) A population-based temporal logic gate for timing and recording chemical events. Molecular Systems Biology 12, 869.

(33) Meysman, P., Sánchez-Rodríguez, A., Fu, Q., Marchal, K., and Engelen, K. (2013) Expression Divergence between Escherichia coli and Salmonella enterica serovar Typhimurium Reflects Their Lifestyles. Molecular Biology and Evolution 30, 1302-1314.

(34) Leventhal, D. S., Sokolovska, A., Li, N., Plescia, C., Kolodziej, S. A., Gallant, C. W., Christmas, R., Gao, J.-R., James, M. J., Abin-Fuentes, A., Momin, M., Bergeron, G., Fisher, A., Miller, P. F., West, K. A., and Lora, J. M. (2020) Immunotherapy with engineered bacteria by targeting the STING pathway for anti-tumor immunity. Nature Communications 11, 2739.

(35) Abedi, M. H., Lee, J., Piraner, D. I., and Shapiro, M. G. (2020) Thermal Control of Engineered T-cells. ACS Synth. Biol. 9, 1941-1950.

(36) Chen, Y.-J., Liu, P., Nielsen, A. A. K., Brophy, J. A. N., Clancy, K., Peterson, T., and Voigt, G. A. (2013) Characterization of 582 natural and synthetic terminators and quantification of their design constraints. Nature Methods 10, 659-664.

(37) Courbet, A., Endy, D., Renard, E., Molina, F., and Bonnet, J. (2015) Detection of pathological biomarkers in human clinical samples via amplifying genetic switches and logic gates. Science Translational Medicine 7, 289ra83-289ra83.

(38) Flynn, J. M., Levchenko, I., Seidel, M., Wickner, S. H., Sauer, R. T., and Baker, T. A. (2001) Overlapping recognition determinants within the ssrA degradation tag allow modulation of proteolysis. PNAS 98, 10584-10589. (39) Roßmanith, J., Weskamp, M., and Narberhaus, F. (2018) Design of a Temperature-Responsive Transcription Terminator. ACS Synth. Biol. 7, 613-621.

(40) Martins, F., Sofiya, L., Sykiotis, G. P., Lamine, F., Maillard, M., Fraga, M., Shabafrouz, K., Ribi, C., Cairoli, A., Guex-Crosier, Y., Kuntzer, T., Michielin, O., Peters, S., Coukos, G., Spertini, F., Thompson, J. A., and Obeid, M. (2019) Adverse effects of immune-checkpoint inhibitors: epidemiology, management and surveillance. Nature Revieres Clinical Oncology 16, 563-580.

(41) Bertrand, A., Kostine, M., Barnetche, T., Truchetet, M.-E., and Schaeverbeke, T. (2015) Immune related adverse events associated with anti-CTLA-4 antibodies: systematic review and meta-analysis. BMC Medicine 13, 211.

(42) Grady, R., and Hayes, F. (2003) Axe-Txe, a broad-spectrum proteic toxin-antitoxin system specified by a multidrugresistant, clinical isolate of Enterococcus faecium. Mol Microbiol 47, 1419-1432.

(43) Fedorec, A. J. H., Ozdemir, T., Doshi, A., Ho, Y.-K., Rosa, L., Rutter, J., Velazquez, O., Pinheiro, V. B., Danino, T., and Barnes, C. P. (2019) Two New Plasmid Post-segregational Killing Mechanisms for the Implementation of Synthetic Gene Networks in Escherichia coli. iScience 14, 323-334.

(44) Chavez, M., Silvestrini, M. T., Ingham, E. S., Fite, B. Z., Mahakian, L. M., Tam, S. M., Ilovitsh, A., Monjazeb, A. M., Murphy, W. J., Hubbard, N. E., Davis, R. R., Tepper, G. G., Borowsky, A. D., and Ferrara, K. W. (2018) Distinct immune signatures in directly treated and distant tumors result from TLR adjuvants and focal ablation. Theranostics 8 , 3611-3628. 
(45) Silvestrini, M. T., Ingham, E. S., Mahakian, L. M., Kheirolomoom, A., Liu, Y., Fite, B. Z., Tam, S. M., Tucci, S. T., Watson, K. D., Wong, A. W., Monjazeb, A. M., Hubbard, N. E., Murphy, W. J., Borowsky, A. D., and Ferrara, K. W. (2017) Priming is key to effective incorporation of image-guided thermal ablation into immunotherapy protocols. $\mathcal{F} C I$ Insight 2.

(46) Landry, B. P., and Tabor, J. J. (2018) Engineering Diagnostic and Therapeutic Gut Bacteria, in Bugs as Drugs, pp 331361. John Wiley \& Sons, Ltd.

(47) Chien, T., Doshi, A., and Danino, T. (2017) Advances in bacterial cancer therapies using synthetic biology. Current Opinion in Systems Biology 5, 1-8.

(48) Dou, J., and Bennett, M. R. (2018) Synthetic Biology and the Gut Microbiome. Biotechnology fournal 13, 1700159.

(49) Riglar, D. T., and Silver, P. A. (2018) Engineering bacteria for diagnostic and therapeutic applications. Nat Rev Microbiol 16, 214-225.

(50) Couture, O., Foley, J., Kassell, N. F., Larrat, B., and Aubry, J.-F. (2014) Review of ultrasound mediated drug delivery for cancer treatment: updates from pre-clinical studies. Translational Cancer Research 3.

(51) Deckers, R., and Moonen, C. T. W. (2010) Ultrasound triggered, image guided, local drug delivery. Fournal of Controlled Release 148, 25-33.

(52) Chowdhury, S., Castro, S., Coker, G., Hinchliffe, T. E., Arpaia, N., and Danino, T. (2019) Programmable bacteria induce durable tumor regression and systemic antitumor immunity. Nature Medicine 25, 1057-1063.

(53) Mimee, M., Citorik, R. J., and Lu, T. K. (2016) Microbiome therapeutics - Advances and challenges. Advanced Drug Delivery Reviewes 105, 44-54.

(54) Voigt, C. A. (2020) Synthetic biology 2020-2030: six commercially-available products that are changing our world. Nature Communications 11, 6379.

(55) González, L. M., Mukhitov, N., and Voigt, C. A. (2020) Resilient living materials built by printing bacterial spores. Nature Chemical Biology 16, 126-133.

(56) Nguyen, P. Q., Courchesne, N.-M. D., Duraj-Thatte, A., Praveschotinunt, P., and Joshi, N. S. (2018) Engineered Living Materials: Prospects and Challenges for Using Biological Systems to Direct the Assembly of Smart Materials. Advanced Materials 30, 1704847.

(57) Gilbert, C., Tang, T.-C., Ott, W., Dorr, B. A., Shaw, W. M., Sun, G. L., Lu, T. K., and Ellis, T. (2021) Living materials with programmable functionalities grown from engineered microbial co-cultures. Nat Mater.

(58) Lee, S. J., Lee, S.-J., and Lee, D.-W. (2013) Design and development of synthetic microbial platform cells for

bioenergy. Front. Microbiol. 4. 